\title{
NUOVI CRITERI PER LA SISTEMAZIONE MAGNETICA
}

\section{Canlo Morelli}

1. Premessa. - - Ie finalita per cui eseguire un rilievo mannetico generale, ciof non derlicato a lini specifici, si possono cosi riassumere: meltere in evidenza il comportamento del campo mannetico terestre (cm.t.), ed individuarne e delimitame le caratleristiche (amomalie) che meglio si prestimo a studiarne le callse; in particolare, a ricararne indicazioni sulla struttura qreologica nascosta e sulla tettonica, nonche piu in dettaglio eventuali dati utili alla prospexione reofisica.

Cio premesso, i chiaro che una campagna mannelica dovri essere preventivamente fissata neqli soppi e nei criteri di esecuzione, e guindi studiata e progettata sulla cartal in particolare: geolovical, onde coorlinarne il programma generale, il numero e la distribuzione delle stazioni. ece. Salvo poi a lasciare alloperatore di adattare tale programma alle esigenze del terreno ed alle necessita che si manifestasero durante l'esecuzione del lavoro.

Seopo della presente relazione $i$ appunto di esporre un nuovo eriterio per l'esecuzione di rilievi magnetici sistematiei, e di precisare in sintesi le norme principali eui attenersi nella compilazione del proframma generale e nell'esecuzione della camplanas. Fondamento sarà l'opportunitie di riture al minimo le misure ascolute, e di sostituirle con mi-mere relative. molto pitu precise rapide. e quindi economiche. Come in eravimetria il pendolo sta cedendo un po' il posto al pravimetro. anche melle operazoni a largo ragrio. eosi nel magneti-mo terrestre i variometri doreblere sotituies per buona parte della campagna ai teodoliti magnetici. con i criteri cle peiù avanti esporremo

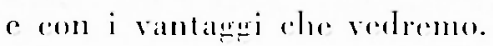

2. Elementi da misurare oprecisione necessaria. - Poiché il c.m.t.

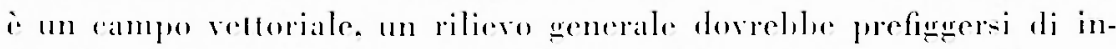
dividuare il rettore del campo in ogni punto in rui si fa stazione. I tal fine i necesentrio misurare tre clementi di questo vettore: ed $i$ generabmente consigliabile misurame l'angolo della declinazione mapnetica $D$. la componente orizzontale $I /$ e la componente verticale $Z$. e ricavare da questi eol calcolo le componenti $X$ e Y secondo un si- 
stema di assi cartesiani con l'asse lelle $x$ rivolto al Nort geografico. l'asse delle y rivolto ad bes le l'asse delle z rivolto verso l'interno della Terat, nonché l'angolo di inclinazione $I$ e l'intensita tolale $F$.

Cio perehé, seguento i criteri che vedremo, si puo raggimgere

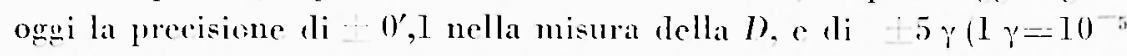
c.g̣.s.) nella misura della $I I$ e della $Z$ : eioe per gli elenenti che meglio consentono di passare all'interpretazione dei risultali. Mentre vieeversa misurando la $I$ e ricavando la $Z$ mediante la formula:

$$
Z=--\Pi \text {. I } I
$$

la precisione risulta di gran lunga minore. Cio si vede subito alifferenziando la formula precerlente:

$$
\left.d Z=d I I \cdot \operatorname{tg} I+I I \cdot(1+\operatorname{tg})^{2} I\right) \cdot d I
$$

Si vede da questa - come del resto i intuitivo -- che alle latitudini mannetiche magrgoriori di $45^{\circ}$, a piceole variazioni della $I$ corrispondono forti variazioni della $Z$, che diventano lanto più cospicue quanto più grande è il valore di $I$.

Passando a valori numeriei e riferendoci all'llalia, arremo $i$ seguenti liniti:

$$
\begin{gathered}
\text { Italia sett. } \quad\left(I=62^{\circ}: I I=21.000()\right. \\
d Z=1.88 . d I I+26,70 d I . \\
\text { Italia merid. }\left(I=52^{\circ} ; I I=26.000\right) \\
d Z=1.28 . d H+19.95 d I .
\end{gathered}
$$

Nelle precellenti, $d I$ va espresso in '.

Ne risulta che per esempio alle latitulini dell'talia selt. un errore di 0 ,5 nella misura della $I$ si riperenote con un errore di hen 13 y nella 7 : ed è noto che nelle misure (assolute) della $I$ ben difficilmente si riesce a garantire il mezzo primo.

La precisione più sopra indicala è quella che oggei si richiede, per le $D, H, Z$ : e vellemo appunto piu aranti $\mid s 3$ ) come si possa ottenerla per questi due ultimi elementi.

Qualora ragioni di economia, o difficolta tecniche, impongano di misurare una sola componente tel c.m.t., sarì senz'altro ta preferirsi la $Z$, sia perché essa più facilmente tlelle altre consente l'interpreta- 
zione dei ristlati, sia perché la conoscenza di questa sola componente permelde ormai (') di calcolare lutte le altre componenti del rampo.

3. Misure assolate, misure relative e distribazione delle stazioni. -.- Prementiano f'oservazione che un rilievo regionale con sole misure rssolute non sarelule piu né convenicnte, per il magrgior tempo e la piu cospicua allrezzalura che queste misure richiedono, né redditizio, in fuanto la lowo precisione polreble essere solo difficilmente guella Jesiderala, e una compensizione piutlosto ardua.

Von conviene d'altra parte neppure effethare misure solo relative, anzitullo perchis per la valutazione del segno delle anomalie esse richiodono di appoggiarsi ad almeno ma misura assoluta; e poi perchí per es. la costante di scala del variometro per la $I I$ ì funzione anclie della 7.

Proponiamo pereio come soluxione di "optimum p per un rilievo revionale, la segruente:

a) ana maslia fondamentale di stazioni assolute (10 ordine) distribute miformemente alla distanza media di cirra $50 \div 150 \mathrm{~km}$ l'mal dall'altra. in modo da rostituire come m reticolato sul quale appogegare le misure relative:

b) man serie di rirruiti ron misnre relative. alla distanza media di circa $10 \div 20$ lim l'una dall'altra (20 ordine), opportunamente distribuite in nodo da collegane in tulli i versi le stazioni del $1^{\circ}$ ordine e da ricoprive muformenente la regione in esame:

c) misare di dellowlio, a distanzal ravicinata $\left(3^{\circ}\right.$ ordine $=$ riliero loralet. ove rio si manifesti opportuno.

Nell: saxioni del l" ordine le mi-ne assolute nei tre elementi I), $I I$ ed $I$ andreblero ripelute un numero di vole tale da garantire una precisione suffeiente nella $D . I I$. e $Z=H$. I $I$. Per esempio, con strumenti rhe diano $D$ entro 0$)^{\prime}, 5$, la misma della $D$ dovehbe essere ripelula almeno 25 vole per rageiungere nella media la precisione di 0 .l: a con strumenti cle diano $I /$ entro $\pm 5 \gamma$ ed $I$ entro + $0^{\prime} .5$. la ripelizione delle misure della $I I$ e deìla $I$, per otlenere il valore della $Z$ ron lapprosimazione di $5 \%$. dovel,be esere per le $|3|$ e $|1|$ precedenti. come dal seguente pospello:

Italia sell. : II non meno di 36 volte:

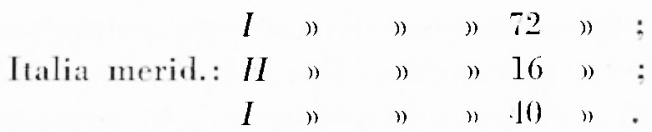


Si vale rosi quanlo sia laboriosia una misura a-soluta suffeientemente presisa tella $Z$. e come sia pereio indisfensabile arlotalre un procerlimento ele consenta di ridure al minimo il numero di fuesele

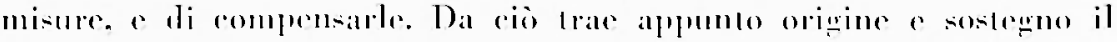
melothe qui proprosto.

Ie stazioni del 2" ardine dovelolere invere essere distribuite in cimenti rhiusi, con partenza od arrivo sulla stessa stazione aseoluta. e comprendenti ommuno almeno un'altra stazione assoluta o allat stazione intermedia più vole rollegala con le misure atsolute. Ie misure

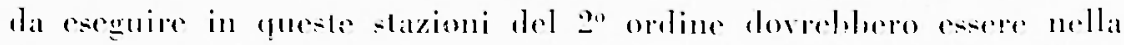

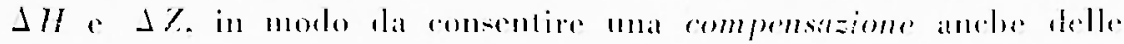

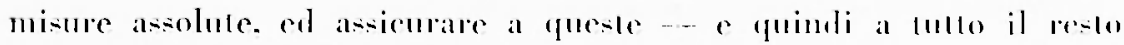

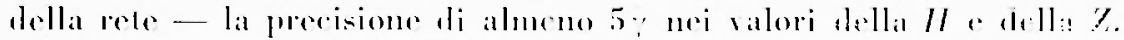

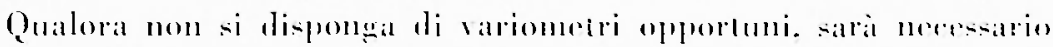

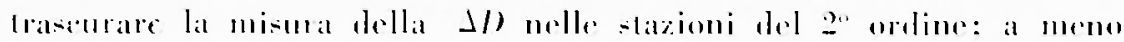

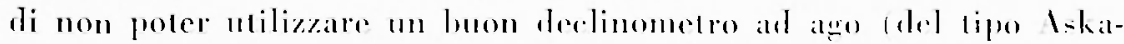

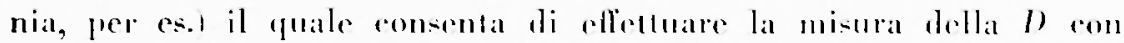

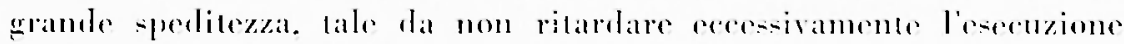
lelle stazioni del $2 "$ oraline.

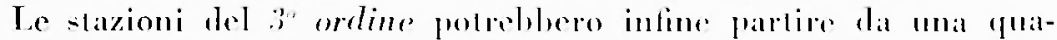
lungue delle stazioni del 20 ordine: anche qui naturalmente i rircuiti

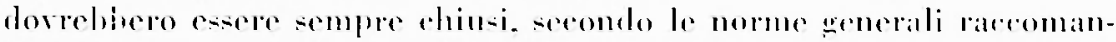
date per le operazinni ron i varomelri.

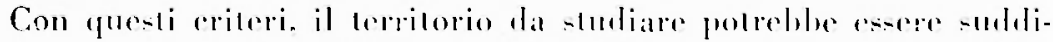

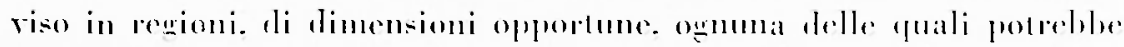

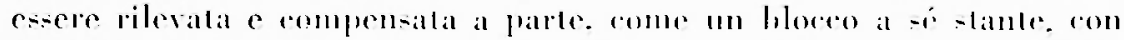
i criteri sopra esposti. Opportumi collewamenti fra una rewione o laltra consentirehbero quindi una compensazione pernerale.

4. Sazione registratrice. - Per lutla la durita della ramplatna.

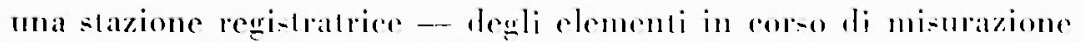

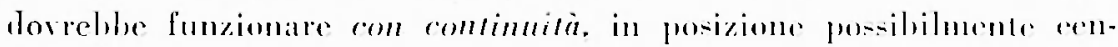
trale ri-pedto alla zona rilevala. Cio allo scopo sopratlutle di formire grli elementi per le ridusioni variazione diurna. mensile. nomele di

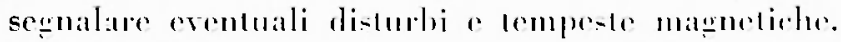

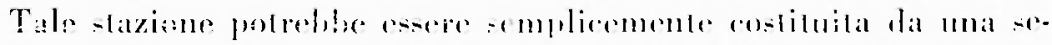

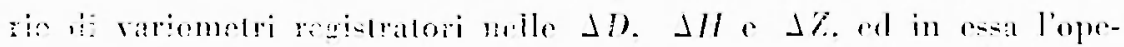

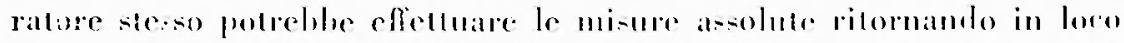


per es. uma vola alla sellimana con gli stumenti fassoluli di camjalgha.

5. Sorlen degli strmmenti. - Da quanto procede pisulat clue per l'esecuzione delle misure assolute oecorrono strumenli che si arici-

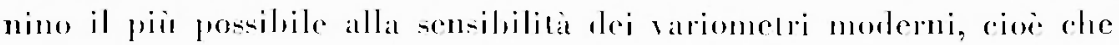

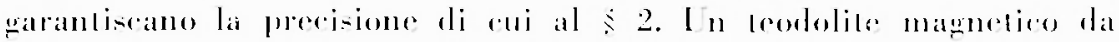

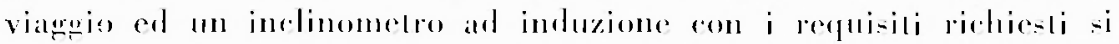

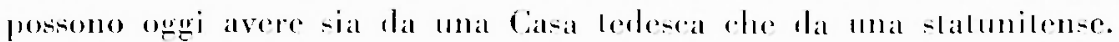

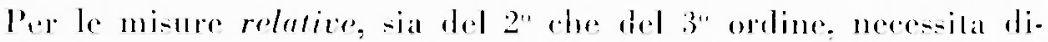
sperre di luoni variometri. Eecellenti ri-ullati hamne lato in questo

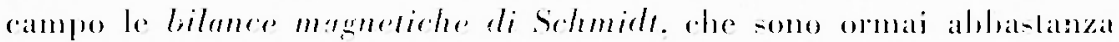

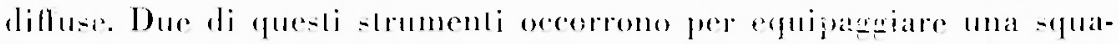

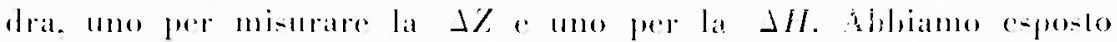
altrove t-f le caralleri-liche e le norme terniehe da seguire con quesli strumenli.

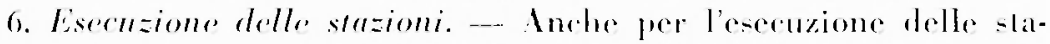
zioni, rimandiano alle nome pia menzionale $\left.\right|^{2}$. Q Qui conviene solo ancora lar presente l'oppertunila ehe in an rilievo regionale ogni ster-

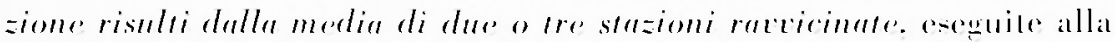

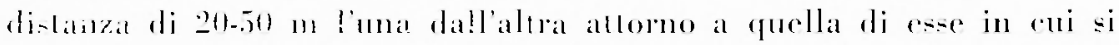

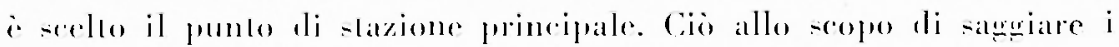
eranlienti del rampo in vare direzioni e di meltere quindi in eridenzar la "normalilion del punlo o la presenza di anomalie. che converri eventualnente precisare con un rilievo del 3 " oreline.

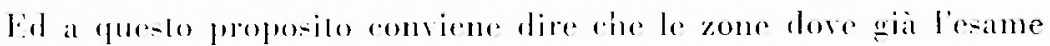
della rarta peologica garanlise la presenza di anomalie irregolari. in

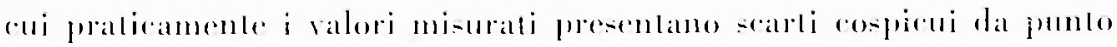

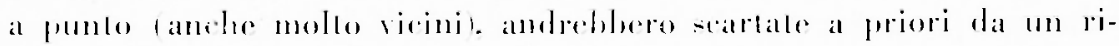

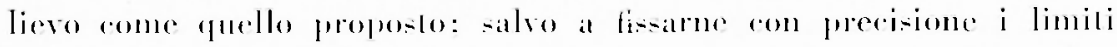
e a studiane in dellatelio qualehe loro porzione quando eic oceorresse. Tali somo ad esempio tulle le zone vulamiche.

Aleune sazioni varionelriche di dellawlo sari poi sempere. ed a maggior ragione. apportumo distribuide allomo alle sazioni del $l^{\text {" }}$ ordinte.

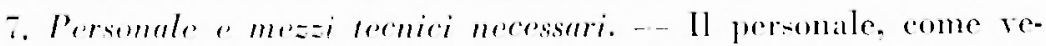

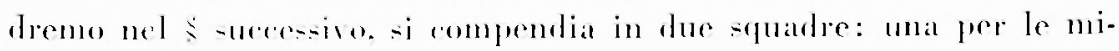
sure atsolule, con un operatore enl un ralcolalores el mal per le mi- 
sure relative ron un operatore, un ealcolatore enl un aintante. Quando essi siano opporlunamente allenati nella suldivisione e nella suecessione dei compili 1-2, la spediterza delle stazioni del 2" e 3" ordine puis anche essere cospicua. Necessita poi naluralmente un automezo, per gli spostamenti rapridi da stazione a stazione: nelle soste l'autista potra pure rendersi utile, per esempio nelle operazioni di contorno.

Fra il rimanente del corredo tecnice, ci sembra qui opportumo ricordare solo: ma piccola haranca smombabile per la stazione regi-

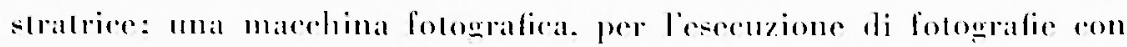
cui corredare la monografia delle stazioni: arte lopografiehe e peologiche: ece. Fentuali: due radio trasmittenti e riecrenti, per il contatto continuo ed immediato lia le due squanlere.

Naturalmente, e per yuanto si i visto, le stazioni del l" ordine andranno essquite con qrandisima cura, e quindi parlare di "speditraza" per queste non avelshe siquificalo.

8. Durata e costo. - Da quanto precerle ir evidente ehe il mageior tempo verra aseorlito per l'esecuzione delle stazioni assolute. Per ognuna di queste infatti le misure dovreblere durare la 3 a 5 geromi, con operabore bene allenato. Prendendo quindi per base un'area di 10.0000 $\mathrm{km}^{-} \mathrm{e}$ supponendo di distrihuire in questat 5 staxioni assolute. lesente zione di queste richiedereblue circa un mese. in assenzal di periodi magnetieamente perturliati.

In questo periodo di tempo la seconda spuadra potreble esenuire in quest'area tutto il rilievo del 2" ardine, parte dei collewamenti con l'area vicina, e anche parte del $3^{\circ}$ ordine.

Qualora la stessa area renise rieoperla solo da misure ascolute anche alla distanza di $20 \mathrm{~km}$ l'una dallaltra. oecorrereblero almono

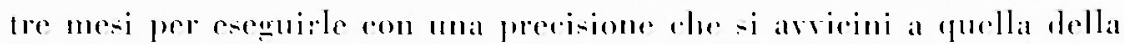
rete qui proposta: e maneherehbe poi ogni posibilita di compensazione, tutto il 2" ordine e lumona parte del $3^{n !}$ Come si velle. anche sulla bise della sola ronvenienza enomomica il rilievo sistematieo di un territorio eon sole mi-ure asolute 2 ormai da considerarsi superalo.

Traducendo in cifre. con i rosti atluali. i dati di cui soprar il rilievo di un'area di $10.0001 \mathrm{~km}^{2}$ eon i eriteri proposti in questa nota vereblue a rostare, considerando solo le spese di campagna del personsle e di consumo o romputamble in T. 15.000 piornalieme. da

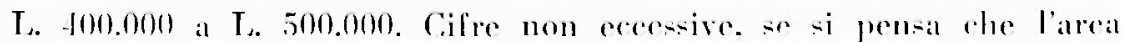
presletla $i$ superiore a quella delle Marehe o lella lemeania.

In conclusione, sembra lecito affermare rite il metorto qui pro- 
posto ofle requisili di precisione, completezza od ecomomia lali da

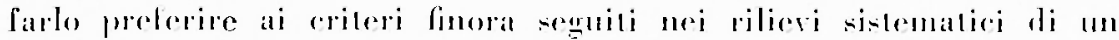
terrilorio.

Islimbo Naz. di Cieofisica - Osserv. di Trieste - Nov. 1919.

\section{RIISTL NTO}

Comsiderale rhe Ir misure magneliche assolute non comsentomo

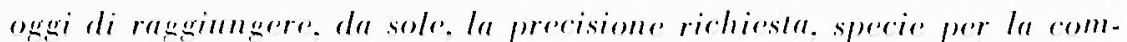

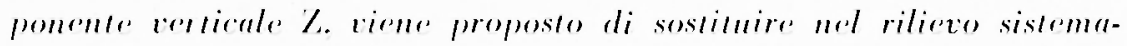
lico di an territorio la maggior parte dello misure assolule con misure relalive. Le misure assolule docreblere costituire solo una rele a lar-

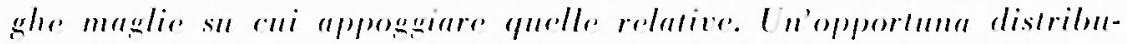
zione di queste consemtirebler la rempensaziome della rele delle mi-

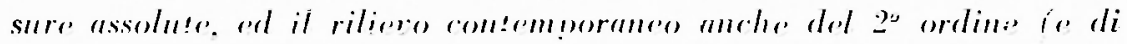

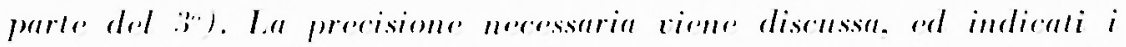

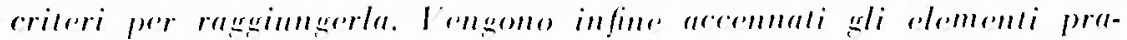
lici ed ecomomici del molodo.

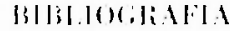

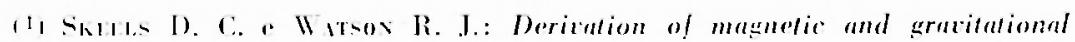
quantinies by surface integraton. Ceophy-ic. MII. 2. 13:-150. april 1919.

(-) Molutu C.: Teoria e pration dei anrometri mannetici da campagna. Yono-

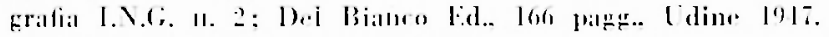

